\title{
Influence of Mixed Cationic-nonionic Surfactant Systems on the Spectral Properties of C.I. Mordant Blue 29 and Its Complexes with Iron(III)
}

\author{
Katarzyna Pytlakowska, Piotr Zerzucha, ${ }^{\dagger}$ and Rozalia Czoik \\ Institute of Chemistry, University of Silesia, Szkolna 9, 40-006 Katowice, Poland
}

\begin{abstract}
Interactions of the anionic form of C.I. Mordant Blue 29 with cationic and nonionic surfactants have been studied by absorption spectroscopy. The dye interacts strongly with oppositely charged surfactants in the pre-micellar concentration range with an accompanying change in its spectral properties, while its interaction with the polyoxyethylene group of nonionic surfactants does not have such remarkable effects. In ternary mixtures, however, the influence of the polyoxyethylene group on the optical properties of C.I. Mordant Blue 29-tetradecyltrimethylammonium bromide and C.I. Mordant Blue 29-octadecyltrimethylammonium chloride systems is quite different. In addition, the influence of the interaction between the $\mathrm{HL}^{3-}$ form of C.I. Mordant Blue 29 and the surfactants on the formation of chelate complexes with iron(III) has been studied. The optimized structures of Fe-Mordant Blue 29 complexes are also reported herein. Geometries have been calculated by the Hartree-Fock method with the cc-pVDZ basis set.
\end{abstract}

(Received July 23, 2010; Accepted March 2, 2011; Published May 10, 2011)

\section{Introduction}

Indicator-surfactant interactions in aqueous systems are central to many research topics due to their wide and varied applications. ${ }^{1-8}$ Studies of such interactions are fundamental in many chemical and pharmaceutical fields, in organic syntheses, and in several industrial applications.

Micellar systems influence the chemical equilibria and the reactivity, as well as the spectral and electrochemical responses of substrates. These properties have proven to be very useful, especially in such areas of analytical chemistry as separation techniques or determinations based on spectral methods. Many analytical applications using micellar solutions involve the determination of metal ions by their complexation with suitable ligands. One of the important classes of such ligands is the hydroxytriphenylmethane group of dyes, which includes C.I. Mordant Blue 29. The analytical possibilities of such ligands can be expanded significantly if the complexation reactions with metal ions are carried out in the presence of ionic or nonionic surfactants or mixtures thereof. The addition of surfactants frequently causes a bathochromic shift of spectral bands and increases the sensitivity of metal analysis through the formation of dye-rich complexes. Metal-to-dye ratios can vary from 1:1 in aqueous solution to $1: 2$ or 1:3 in surfactant-containing solutions. Such effects are observed if the molar excess of the cationic surfactant over the chromogenic reagent varies between 5- and 15 -fold. The extent of this range depends on the nature of the surfactant used (among other factors, on the critical micelle concentration (CMC) of cationic surfactants). ${ }^{9}$ In the case of nonionic surfactants, spectral changes are only observed at

$\dagger$ To whom correspondence should be addressed.

E-mail: piotr.zerzucha@us.edu.pl concentrations exceeding the CMC. ${ }^{10}$ However, the mechanism of micellar effects on the formation of analytically useful complexes is still not well understood.

Today, C.I. Mordant Blue 29 (Fig. 1) is probably the most widely used chromogenic reagent of the hydroxytriphenylmethane dyes. The dye has pronounced chelating properties due to the presence of donor groups such as $-\mathrm{COOH},-\mathrm{OH}$, and $>\mathrm{C}=\mathrm{O}$. Additionally, the sulfonic group on the third ring of C.I. Mordant Blue 29 is responsible for the solubility of the dye and its complexes in water.

Being an excellent chelating reagent, Mordant Blue 29 is used for the quantitative and qualitative determination of a variety of metal ions. The reagent is sensitive but non-selective, and reacts with many metal ions in weakly acidic or nearly neutral media to form red, blue, or green water-soluble complexes according to the $\mathrm{pH}$ value of the solution. C.I. Mordant Blue 29 forms complexes with over 30 metal ions, including $\mathrm{Be}(\mathrm{II}), \mathrm{Mg}(\mathrm{II})$,<smiles>CC1=CC(=C(c2cc(C)c(O)c(C(=O)O)c2)c2c(Cl)ccc(S(=O)(=O)O)c2Cl)C=C(C(=O)O)C1=O</smiles>

Fig. 1 The chemical structure of C.I. Mordant Blue 29. 


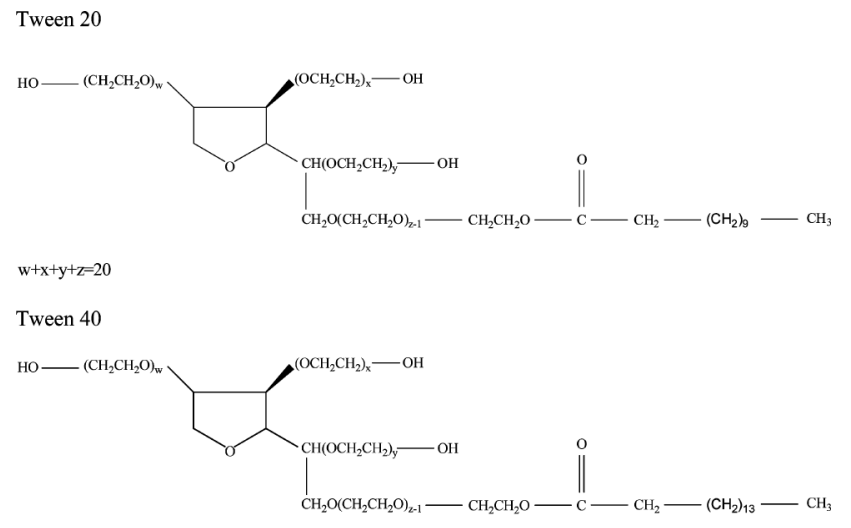

$w+x+y+z=20$

Tween 60

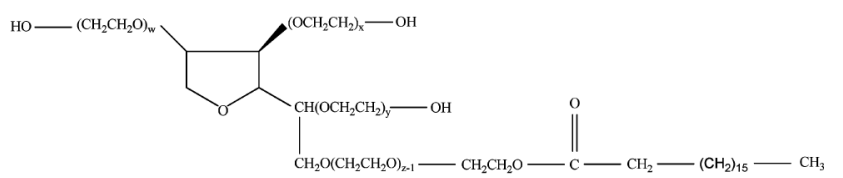

$\mathrm{w}+\mathrm{x}+\mathrm{y}+\mathrm{z}=20$
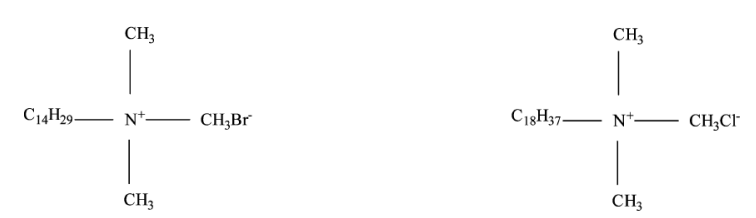

TTA

ODTA

Fig. 2 The chemical structures of TTA, ODTA, Tween 20, Tween 40 , and Tween 60 .

$\mathrm{Al}(\mathrm{III}), \mathrm{In}(\mathrm{III}), \mathrm{Cu}(\mathrm{II}), \mathrm{Cd}(\mathrm{II}), \mathrm{Ti}(\mathrm{IV}), \mathrm{Zr}(\mathrm{IV}), \mathrm{Hf}(\mathrm{IV}), \mathrm{Fe}(\mathrm{III})$, $\mathrm{Pd}(\mathrm{II}), \mathrm{Sc}(\mathrm{III}), \mathrm{Y}(\mathrm{III}), \mathrm{Th}(\mathrm{IV})$, and U(VI), and these compounds are used in spectrophotometric methods. ${ }^{11}$

The aim of the work described herein has been to study the influence of cationic and/or nonionic surfactants on the spectral characteristics of C.I. Mordant Blue 29 and its complexes with iron(III) by means of spectrophotometric measurements.

\section{Experimental}

\section{Chemicals}

C.I. Mordant Blue 29 (Chromazurol S, CAS) $\left(1 \times 10^{-3} \mathrm{~mol} \mathrm{~L}^{-1}\right)$ was a commercial product from Merck; tetradecyltrimethylammonium bromide (TTA) $\left(1 \times 10^{-2} \mathrm{~mol} \mathrm{~L}^{-1}\right)$, octadecyltrimethylammonium chloride (ODTA) $\left(1 \times 10^{-2} \mathrm{~mol} \mathrm{~L}^{-1}\right)$, polyoxyethylene sorbian-monolaurate (Tween 20) (1\%), polyoxyethylene sorbian-monopalmitate (Tween 40$)(1 \%)$, and polyoxyethylene sorbian-monostearate (Tween 60) (1\%) were commercial products from Fluka. C.I. Mordant Blue 29 was purified by repeated recrystallization from aqueous acetone solution. ${ }^{12}$ The surfactants were used as obtained, without further purification. A standard solution of iron(III) $\left(1 \mathrm{mg} \mathrm{mL}^{-1}\right)$, was obtained from Merck. The phthalate buffer solutions of $\mathrm{pH}$ 5.56, 6.0, and 6.2 were prepared from sodium hydroxide (POCh) and potassium hydrogenphthalate (POCh). The phosphate buffer solution of $\mathrm{pH} 6.0$ was prepared from potassium dihydrogenphosphate (POCh) and sodium hydrogenphosphate (POCh). The $\mathrm{pH}$ values of the buffer solutions were controlled

$$
\begin{aligned}
& \mathrm{pKa}_{1}=-2.2 \quad \mathrm{pKa}_{2}=-1.2 \quad \mathrm{pKa}_{3}=2.25 \quad \mathrm{pKa}_{4}=4.88 \quad \mathrm{pKa}_{5}=11.75
\end{aligned}
$$

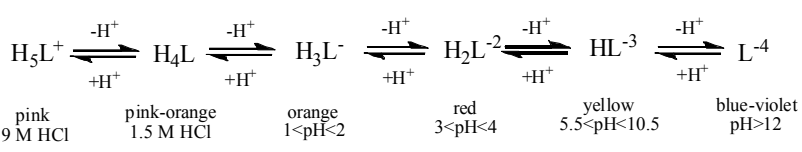

Fig. 3 The schematic diagram of dissociation in stages of C.I. Mordant Blue 29. ${ }^{17,18}$

by $\mathrm{pH}$-meter measurements. All solutions were prepared in doubly-distilled water. The chemical structures of the surfactants used in this work are presented in Fig. 2.

\section{Measurements}

Absorption measurements were performed on a Jasco V-530 spectrophotometer (Jasco, Japan) using solutions in $1 \mathrm{~cm}$ cuvettes. UV/Vis spectra were recorded over the wavelength range from 350 to $700 \mathrm{~nm}$. All $\mathrm{pH}$ measurements were made using a CP-315 pH-meter (Elmetron) with a combined glass electrode.

\section{Computational details}

The molecular structures of $\mathrm{FeL}, \mathrm{FeL}_{2}$, and $\mathrm{FeL}_{3}$ complexes in the ground state were optimized by the Hartree-Fock method ${ }^{13,14}$ using the cc-pVDZ basis set. ${ }^{15}$ The optimized geometries of the complexes were verified by performing frequency calculations. All of the vibrations in the calculated vibrational spectra of the complexes were real, thus indicating that the optimized geometries corresponded to true energy minima. The Gaussian 09 program ${ }^{16}$ was used for the calculations.

\section{Results and Discussion}

C.I. Mordant Blue 29 is a polybasic acid $\left(\mathrm{H}_{4} \mathrm{In}\right)$, and it exists in five different structural prototropic forms according to the $\mathrm{pH}$ of the solution $\left(\mathrm{H}_{5} \mathrm{In}^{+}, \mathrm{H}_{3} \mathrm{In}^{-}, \mathrm{H}_{2} \mathrm{In}^{2-}, \mathrm{HIn}^{3-}, \mathrm{In}^{4-}\right)$. The color of these charged species is found to change with variations in the hydrogen ion concentration. A schematic diagram of the dissociation stages of C.I. Mordant Blue 29 is shown in Fig. 3.

At pH 6.0 - 6.2, as used in these studies, C.I. Mordant Blue 29 exists mainly in the $\mathrm{HL}^{3-}$ form, which corresponds to a species having two dissociated carboxyl groups. In a buffered aqueous solution, the spectrum of C.I. Mordant Blue 29 corresponding to the $\mathrm{HL}^{3-}$ species (Fig. 3) shows two bands: a shorter wavelength band at around $430 \mathrm{~nm}$ and a longer wavelength band at around $545 \mathrm{~nm}$. In the presence of cationic surfactants, spectacular changes are seen in the electronic absorption spectrum of C.I. Mordant Blue 29 (Fig. 4). These are related to interactions of the oppositely charged ionic dye and surfactant, which are governed by attractive long- and short-range forces, such as electrostatic interactions between ions, dispersive interactions, charge-transfer interactions between the quaternary ammonium cation of TTA or ODTA and the $\pi$-electron system of the C.I. Mordant Blue 29, and hydrophobic interactions. ${ }^{19,20}$

The addition of a cationic surfactant to the anionic form of C.I. Mordant Blue 29 causes a change in the shapes of its absorption bands as well as in its sensitivity. The shorter wavelength band, appearing at $430 \mathrm{~nm}$, does not change position, but its absorbance is decreased. For the absorption peak at $545 \mathrm{~nm}$, both a large bathochromic shift $(\Delta \lambda=80 \mathrm{~nm})$ and a hyperchromic effect are observed. The absorbance increases with increasing length of the hydrocarbon chain of the 


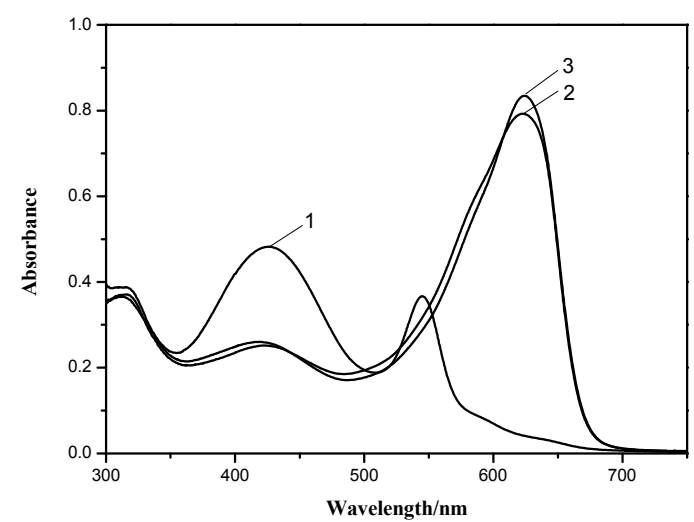

Fig. 4 Absorption spectra of: 1, C.I. Mordant Blue 29; 2, C.I. Mordant Blue 29-TTA; 3, C.I. Mordant Blue 29-ODTA. $c_{\text {C.I.MordantBlue29, }}$ $4 \times 10^{-5} \mathrm{~mol} \mathrm{~L}^{-1} ; c_{\text {ODTA }}, 4 \times 10^{-4} \mathrm{~mol} \mathrm{~L}^{-1} ; c_{\mathrm{TTA}}, 4 \times 10^{-4} \mathrm{~mol} \mathrm{~L}^{-1} ; \mathrm{pH}$, $6.2 ; 1=1 \mathrm{~cm}$

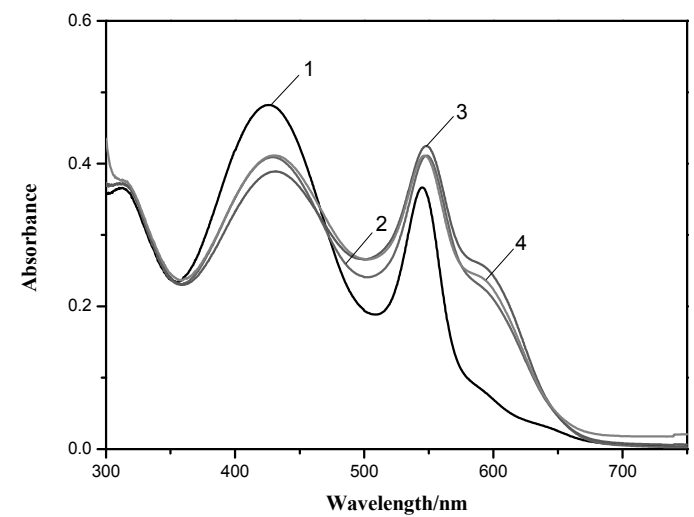

Fig. 5 Absorption spectra of: 1, C.I. Mordant Blue 29; 2, C.I. Mordant Blue 29-Tween 20; 3, C.I. Mordant Blue 29-Tween 40; 4, C.I. Mordant Blue 29-Tween 60. $c_{\text {C.I.MordantBlue29, }} 4 \times 10^{-5} \mathrm{~mol} \mathrm{~L}^{-1} ; c_{\text {Tween20, }}$, $2 \times 10^{-1} \% ; c_{\text {Tween } 40}, 2 \times 10^{-1} \% ; c_{\text {Tween } 60}, 2 \times 10^{-1} \% ; \mathrm{pH}, 6.2 ; 1=1 \mathrm{~cm}$.

cationic surfactant. This means that the ODTA cation interacts more strongly with C.I. Mordant Blue 29 than does the TTA cation.

The Tweens group of nonionic surfactants does not have such remarkable effects on the spectral properties of C.I. Mordant Blue 29, but they do change its molar absorptivity (Fig. 5). Changes in absorbance can be explained in terms of hydrogen bonding, short-range dispersive forces, and hydrophobic interactions between the anionic form of C.I. Mordant Blue 29 and the nonionic surfactant. ${ }^{21}$ The nonlinear variations in the absorbances induced by the Tweens suggest the influence of factors other than the length of the nonpolar tail of the nonionic surfactant. On the other hand, these interactions are not strong enough to change the position of the absorption peaks.

Results obtained for ternary mixtures show that the influences of the Tweens group on the optical properties of C.I. Mordant Blue 29-TTA (Fig. 6) and C.I. Mordant Blue 29-ODTA (Fig. 7) systems are quite different. For the C.I. Mordant Blue 29-TTA system, the absorbance increases with increasing number of the Tween, i.e. with increasing length of the nonpolar tail of the nonionic surfactant, while for the C.I. Mordant Blue 29-ODTA system the inverse tendency is observed. The interaction between the anionic form of the dye and the mixture of cationic

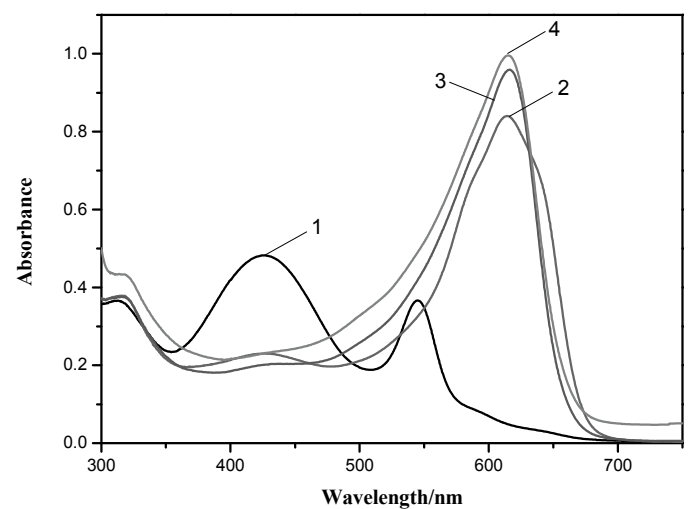

Fig. 6 Absorption spectra of: 1, C.I. Mordant Blue 29; 2, C.I. Mordant Blue 29-TTA-Tween 20; 3, C.I. Mordant Blue 29-TTA-Tween 40; 4, C.I. Mordant Blue 29-TTA-Tween 60; $c_{\text {C.I.MordantBlue29, }}$ $4 \times 10^{-5} \mathrm{~mol} \mathrm{~L}^{-1} ; c_{\mathrm{TTA}}, 4 \times 10^{-4} \mathrm{~mol} \mathrm{~L}^{-1} ; c_{\text {Tween } 20}, 2 \times 10^{-1} \% ; c_{\text {Tween } 40}$, $2 \times 10^{-1} \% ; c_{\text {Tween } 60}, 2 \times 10^{-1} \% ; \mathrm{pH}, 6.2 ; 1=1 \mathrm{~cm}$.

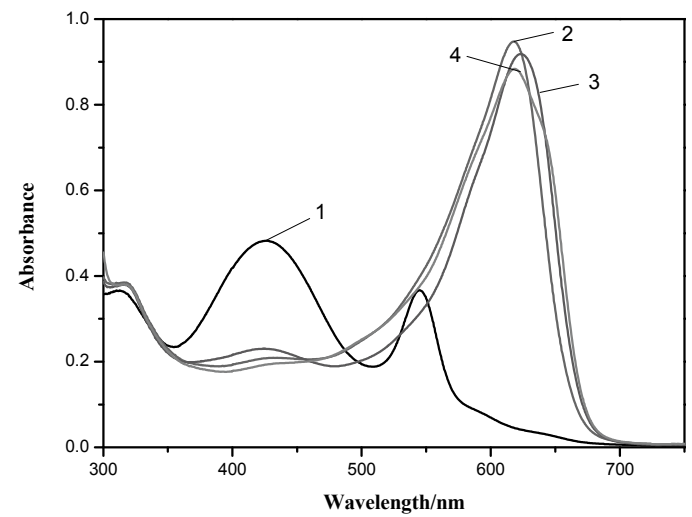

Fig. 7 Absorption spectra of: 1, C.I. Mordant Blue 29; 2, C.I. Mordant Blue 29-ODTA-Tween 20; 3, C.I. Mordant Blue 29-ODTA-Tween 40; 4, C.I. Mordant Blue 29-ODTA-Tween 60; $c_{\text {C.I.MordantBlue29, }} \quad 4 \times 10^{-5} \mathrm{~mol} \mathrm{~L}^{-1} ; \quad c_{\text {ODTA }}, \quad 4 \times 10^{-4} \mathrm{~mol} \mathrm{~L}^{-1} ; \quad c_{\text {Tween20 }}$, $2 \times 10^{-1} \% ; c_{\text {Tween } 40}, 2 \times 10^{-1} \% ; c_{\text {Tween60 }}, 2 \times 10^{-1} \% ; \mathrm{pH}, 6.2 ; 1=1 \mathrm{~cm}$.

and nonionic surfactants depends on the chemical structures of the surfactants. In binary systems with TTA or ODTA, the interaction depends on their hydrophobicity. In ternary systems, the addition of the nonionic surfactant changes the strength of the interactions between the cationic surfactant and C.I. Mordant Blue 29. Comparing the chemical formulae of the Tweens (Fig. 2), one can see that they differ from each other in the hydrophobic alkyl group. The longer the polyoxyethylene group, the larger the partial negative charge on the oxygen bridges, which allows stronger charge-transfer interactions between the long-chain hydrophobic group of the ODTA surfactant and the micelles of the Tween. Stronger interactions between the cationic and nonionic surfactants lead to a decrease in the strength of the interactions between the cationic surfactant and the anionic dye, which is manifested as a decrease in the molar absorptivities of ternary systems with ODTA in comparison with the systems with TTA. ${ }^{5}$ Furthermore, a small hypsochromic shift $(\Delta \lambda=7 \mathrm{~nm})$ is also observed in each of the studied ternary systems in comparison to shift in the binary one.

The interactions between the $\mathrm{HL}^{3-}$ form of C.I. Mordant Blue 29 and the surfactants influence the formation of chelate complexes with iron(III). Investigations on ternary systems 
Table 1 The optimum determination conditions of iron(III) complexes with C.I. Mordant Blue 29 in the presence of TTA and Tweens

\begin{tabular}{|c|c|c|c|c|c|c|c|c|}
\hline \multirow{2}{*}{ Studied system } & \multirow{2}{*}{$\lambda_{\max } / \mathrm{nm}$} & \multirow{2}{*}{$\mathrm{pH}$} & \multirow{2}{*}{$\begin{array}{l}\text { Time required to } \\
\text { achieve equilibrium } \\
\text { state/min }\end{array}$} & \multirow{2}{*}{ Order of reagent addition } & \multicolumn{3}{|c|}{ Minimal molar excess } & \multirow{2}{*}{$\begin{array}{l}\text { Stoichiometry of the } \\
\text { examined complexes in } \\
\text { the presence of SAS }\end{array}$} \\
\hline & & & & & CAS & TTA & Tween $^{\mathrm{a}}$ & \\
\hline Fe-CAS-TTA & 674.0 & 6.0 & 10 & $\begin{array}{l}\text { Fe(III)-TTA-CAS- } \\
\text { phosphate buffer }\end{array}$ & 4.3 & 38 & - & $1: 2$ \\
\hline Fe-CAS-TTA-Tween 20 & 643.0 & 6.2 & 5 & $\begin{array}{l}\text { Fe(III)-CAS-Tween 20- } \\
\text { TTA-phthalate buffer }\end{array}$ & 3.6 & 48 & $4.3 \times 10^{3}$ & $1: 3$ \\
\hline Fe-CAS-TTA-Tween 40 & 641.5 & 6.2 & 40 & $\begin{array}{l}\text { Fe(III)-Tween 40-CAS- } \\
\text { TTA-phthalate buffer }\end{array}$ & 4.2 & 56 & $5.0 \times 10^{3}$ & $1: 3$ \\
\hline Fe-CAS-TTA-Tween 60 & 636.0 & 6.0 & 50 & $\begin{array}{l}\mathrm{Fe} \text { (III)-TTA-CAS-Tween } \\
\text { 60-phthalate buffer }\end{array}$ & 5.6 & 67 & $6.0 \times 10^{3}$ & $1: 4$ \\
\hline
\end{tabular}

a. Minimal weight excess amounts of the Tweens over Fe(III)-molecular masses of Tween 40 and Tween 60 nonionic surfactants are unknown. b. SAS, surface active substances.

Table 2 The optimum determination conditions of iron(III) complexes with CAS in the presence of ODTA and Tweens

\begin{tabular}{|c|c|c|c|c|c|c|c|c|}
\hline \multirow{2}{*}{ Studied system } & \multirow{2}{*}{$\lambda_{\max } / \mathrm{nm}$} & \multirow{2}{*}{$\mathrm{pH}$} & \multirow{2}{*}{$\begin{array}{l}\text { Time required to } \\
\text { achieve equilibrium } \\
\text { state/min }\end{array}$} & \multirow{2}{*}{ Order of reagent addition } & \multicolumn{3}{|c|}{ Minimal molar excess } & \multirow{2}{*}{$\begin{array}{l}\text { Stoichiometry of the } \\
\text { examined complexes in } \\
\text { the presence of SAS }\end{array}$} \\
\hline & & & & & CAS & TTA & Tween $^{a}$ & \\
\hline Fe-CAS-ODTA & 667.0 & 5.56 & 5 & $\begin{array}{l}\text { Fe(III)-CAS-ODTA } \\
\text { phthalate buffer }\end{array}$ & 3.2 & 44 & - & $1: 2$ \\
\hline Fe-CAS-ODTA-Tween 20 & 664.0 & 6.2 & 5 & $\begin{array}{l}\text { Fe(III)-CAS-Tween 20- } \\
\text { ODTA-phthalate buffer }\end{array}$ & 5.2 & 52 & $3.6 \times 10^{3}$ & $1: 3$ \\
\hline Fe-CAS- ODTA-Tween 40 & 663.0 & 6.2 & 25 & $\begin{array}{l}\text { Fe(III)-Tween 40-ODTA- } \\
\text { CAS-phthalate buffer }\end{array}$ & 4.4 & 52 & $4.2 \times 10^{3}$ & $1: 3$ \\
\hline Fe-CAS- ODTA-Tween 60 & 662.0 & 6.0 & 5 & $\begin{array}{l}\text { Fe(III)-Tween 60-CAS- } \\
\text { ODTA-phthalate buffer }\end{array}$ & 4.8 & 40 & $3.8 \times 10^{3}$ & $1: 3$ \\
\hline
\end{tabular}

a. Minimal weight excess amounts of the Tweens over Fe(III)-molecular masses of Tween 40 and Tween 60 nonionic surfactants are unknown. b. SAS, surface active substances.

with iron(III) were performed in the analytical range, ${ }^{9}$ i.e. with a molar excess of cationic surfactant over chromogenic reagent of between 5 and 15 (below the critical micelle concentration, $\mathrm{CMC}$ ). In this range, the absorbances of ternary systems are maximized and are independent of increasing concentration of cationic surfactant, while species are solubilized by submicellar surfactant aggregates. Studies on quaternary systems were carried out in the same analytical range for the cationic surfactant and above the CMC in the case of nonionic surfactants.

C.I. Mordant Blue 29 forms a number of complexes with compositions $\mathrm{Fe}_{2} \mathrm{~L}$, FeLH, $\mathrm{Fe}_{2} \mathrm{~L}_{2}$, and $\mathrm{FeL}_{2},{ }^{22} \mathrm{Fe}_{2} \mathrm{~L}, \mathrm{FeL}, \mathrm{FeL}_{2}$, and $\mathrm{FeL}_{4}$, or $\mathrm{Fe}_{2} \mathrm{~L}$, FeLH, $\mathrm{FeL}_{2}$, and $\mathrm{FeL}_{3}{ }^{23}$ depending on the acidity and concentration. The reagent and its complexes are readily soluble in water and are sufficiently stable. The molar absorption coefficients of binary chelate Fe-C.I. Mordant Blue 29 are in the range $0.43 \times 10^{5}-0.71 \times 10^{5} \mathrm{~L} \mathrm{~mol}^{-1} \mathrm{~cm}^{-1}{ }^{23,24} \mathrm{~A}$ marked increase in the sensitivity and color contrast for the reaction of iron(III) with C.I. Mordant Blue 29 can be attained through the formation of complexes in the presence of surfactants. To establish optimum conditions for the formation of $\mathrm{Fe}(\mathrm{III})$-C.I. Mordant Blue 29 chelate in the presence of surfactants, we examined the influences of $\mathrm{pH}$ and C.I. Mordant Blue 29, TTA/ODTA, and Tween concentrations on the measured absorbance. The conditions required for the studied systems are given in Tables 1 and 2. The complexes proved to be stable for at least $2 \mathrm{~h}$. The spectral characteristics of the complexes are shown in Table 3.

Investigations of ternary and quaternary complexes of iron(III) with C.I. Mordant Blue 29 showed that, in the presence of the surfactants, higher molar absorption coefficients (Table 3) are obtained compared with the corresponding binary chelate Fe-C.I. Mordant Blue $29 \quad\left(\varepsilon=0.43 \times 10^{5}-0.71 \times\right.$ $\left.10^{5} \mathrm{~L} \mathrm{~mol}^{-1} \mathrm{~cm}^{-1}\right)$. This increase in the molar absorption coefficient is similar to the marked increase in the $\varepsilon$ value seen for the completely deprotonated acid-base form of the reagent, involving complete delocalization of the electron pair of the phenolic oxygen with formation of the completely symmetrical reagent anion $\mathrm{L}^{4-}\left(\varepsilon=7.56 \times 10^{4} \mathrm{~L} \mathrm{~mol}^{-1} \mathrm{~cm}^{-1}\right)$ compared with the values for the other acid-base forms $\left(\varepsilon=2.24 \times 10^{4}-2.30 \times\right.$ $\left.10^{4} \mathrm{~L} \mathrm{~mol}^{-1} \mathrm{~cm}^{-1}\right){ }^{21} \quad$ Furthermore, the increase in the molar absorption coefficient is also connected with the composition of the formed complexes. In the absence of surfactants, iron(III) reacts with C.I. Mordant Blue 29 to form a complex with a 1:1 molar ratio. In the presence of surfactants, the increase in the number of C.I. Mordant Blue 29 ions in the structures of the chelates results in the formation of complexes with a 1:2 molar ratio for the ternary systems and a 1:3 or 1:4 molar ratio for the quaternary systems. This fact can be explained in terms of reversal of the metal ion hydrolysis in the presence of surfactants. This process promotes the coordination of more than one dye anion to the metal ion. The marked bathochromic shift can likewise be explained by the higher degree of delocalization of the electron pair of the oxygen into the $\pi$-electron system of the ternary and quaternary complexes as compared with the delocalization in the reagent anion $\mathrm{L}^{4}$.

It is not possible to obtain definitive information about the structure of the chelate based on the experimental results described in this paper. The chelation may either occur between the quinoid oxygen and the adjacent carboxylic oxygen, or the metal ion may coordinate between the phenolic oxygen and the 
Table 3 Spectrophotometric characteristic of iron(III) complexes with CAS in the presence of TTA/ODTA and Tweens

\begin{tabular}{|c|c|c|c|c|}
\hline Parameter & Fe-CAS-TTA & Fe-CAS-TTA-Tween 20 & Fe-CAS-TTA-Tween 40 & Fe-CAS-TTA-Tween 60 \\
\hline Molar absorptivity, $\varepsilon / \mathrm{L} \mathrm{mol}^{-1} \mathrm{~cm}^{-1}$ & $(1.07 \pm 0.04) \times 10^{5}$ & $(1.48 \pm 0.03) \times 10^{5}$ & $(2.07 \pm 0.04) \times 10^{5}$ & $(2.25 \pm 0.05) \times 10^{5}$ \\
\hline 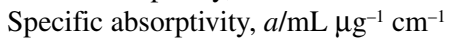 & $1.92 \pm 0.07$ & $2.64 \pm 0.06$ & $3.71 \pm 0.08$ & $4.02 \pm 0.10$ \\
\hline Sandell's sensitivity, $S / \mu \mathrm{g} \mathrm{cm}^{-2}$ & $(5.20 \pm 0.19) \times 10^{-4}$ & $(3.79 \pm 0.08) \times 10^{-4}$ & $(2.70 \pm 0.05) \times 10^{-4}$ & $(2.49 \pm 0.06) \times 10^{-4}$ \\
\hline Detection limit $/ \mu \mathrm{g} \mathrm{Fe} \mathrm{mL}^{-1}$ & $7.2 \times 10^{-3}$ & $5.30 \times 10^{-4}$ & $4.63 \times 10^{-4}$ & $4.27 \times 10^{-4}$ \\
\hline Limit of quantification $/ \mu \mathrm{g} \mathrm{Fe} \mathrm{mL}^{-1}$ & $2.4 \times 10^{-3}$ & $1.59 \times 10^{-3}$ & $1.39 \times 10^{-3}$ & $1.28 \times 10^{-3}$ \\
\hline \multirow[t]{2}{*}{ 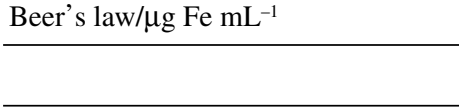 } & $0.08-0.48$ & $0.08-0.56$ & $0.04-0.48$ & $0.04-0.40$ \\
\hline & Fe-CAS-ODTA & Fe-CAS-ODTA-Tween 20 & Fe-CAS-ODTA-Tween 40 & Fe-CAS-ODTA-Tween 60 \\
\hline Molar absorptivity, $\varepsilon / \mathrm{L} \mathrm{mol}^{-1} \mathrm{~cm}^{-1}$ & $(9.38 \pm 0.23) \times 10^{4}$ & $(1.62 \pm 0.06) \times 10^{5}$ & $(1.50 \pm 0.01) \times 10^{5}$ & $(1.35 \pm 0.01) \times 10^{5}$ \\
\hline 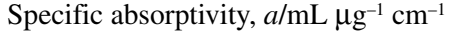 & $1.68 \pm 0.04$ & $2.89 \pm 0.10$ & $2.67 \pm 0.02$ & $2.41 \pm 0.01$ \\
\hline Sandell's sensitivity, $S / \mu \mathrm{g} \mathrm{cm}^{-2}$ & $(5.96 \pm 0.15) \times 10^{-4}$ & $(3.47 \pm 0.13) \times 10^{-4}$ & $(3.82 \pm 0.13) \times 10^{-4}$ & $(4.17 \pm 0.05) \times 10^{-4}$ \\
\hline Detection limit $/ \mu \mathrm{g} \mathrm{Fe} \mathrm{mL}^{-1}$ & $2.07 \times 10^{-3}$ & $3.55 \times 10^{-4}$ & $4.33 \times 10^{-4}$ & $6.11 \times 10^{-4}$ \\
\hline Limit of quantification $/ \mu \mathrm{g} \mathrm{Fe} \mathrm{mL}^{-1}$ & $6.22 \times 10^{-3}$ & $1.07 \times 10^{-3}$ & $1.30 \times 10^{-3}$ & $1.83 \times 10^{-3}$ \\
\hline 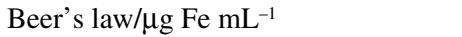 & $0.08-0.56$ & $0.08-0.56$ & $0.08-0.48$ & $0.08-0.48$ \\
\hline
\end{tabular}

adjacent carboxylic oxygen. It is interesting to note that the $\lambda_{\max }$ of the studied chelates is in the range $636-674 \mathrm{~nm}$, whereas the $\lambda_{\max }$ of C.I. Mordant Blue 29 is at $600.5 \mathrm{~nm}$ in a strongly alkaline medium and at $426.5 \mathrm{~nm}$ in weakly acidic or nearly neutral media. It is evident that $\lambda_{\max }$ of the complexes is closer to that of the reagent in an alkaline medium. This effect is connected with dissociation of the proton from the phenolic group, enabling more complete delocalization of the electron pair of the oxygen into the $\pi$-electron system of the reagent molecule. Taking this into account, we suggest that, during chelation, the metal ion coordinates between the phenolic oxygen and the adjacent carboxylic oxygen. Our assumptions concerning the structure of the Fe-C.I. Mordant Blue 29 complexes were confirmed by means of theoretical calculations. The geometrical structures of the complexes were optimized by employing the Hartree-Fock method with the cc-pVDZ basis set. The molecular structures of Fe-C.I. Mordant Blue 29 complexes with the compositions $\mathrm{FeL}, \mathrm{FeL}_{2}$, and $\mathrm{FeL}_{3}$ are shown in Fig. 8.

\section{Conclusions}

The dye-surfactant and metal ion-dye-surfactant interactions in binary, ternary, and, in the case of iron(III) complexes, also in quaternary mixtures, are discussed on the basis of the results of spectrophotometric measurements. In the studied systems, the chemical structure of the anionic form of the dye, as well as the structures of the cationic and nonionic surfactants, affects their interactions. For binary systems, C.I. Mordant Blue 29-TTA and C.I. Mordant Blue 29-ODTA, the hydrophobicity of the alkyl chain, which is responsible for hydrophobic interactions, as well as the electrostatic attraction between the oppositely charged ions, play important roles in the dye-surfactant interactions. In binary systems with surfactants of the Tweens group, short-range dispersive forces and hydrophobic interactions between the anionic dye and nonionic surfactant play a dominant role, but the absorbance varies nonlinearly with increasing length of the nonpolar tail of the nonionic surfactant. In ternary systems, C.I. Mordant Blue 29-TTA-Tween and C.I. Mordant Blue 29-ODTA-Tween, the spectral properties of C.I. Mordant Blue 29 depend both on the chemical structure of the anionic form of the dye and on the structures of the surfactants. It has been observed that the longer the polyoxyethylene group, the larger the partial negative charge on the oxygen bridges,
$\mathrm{FeL}$
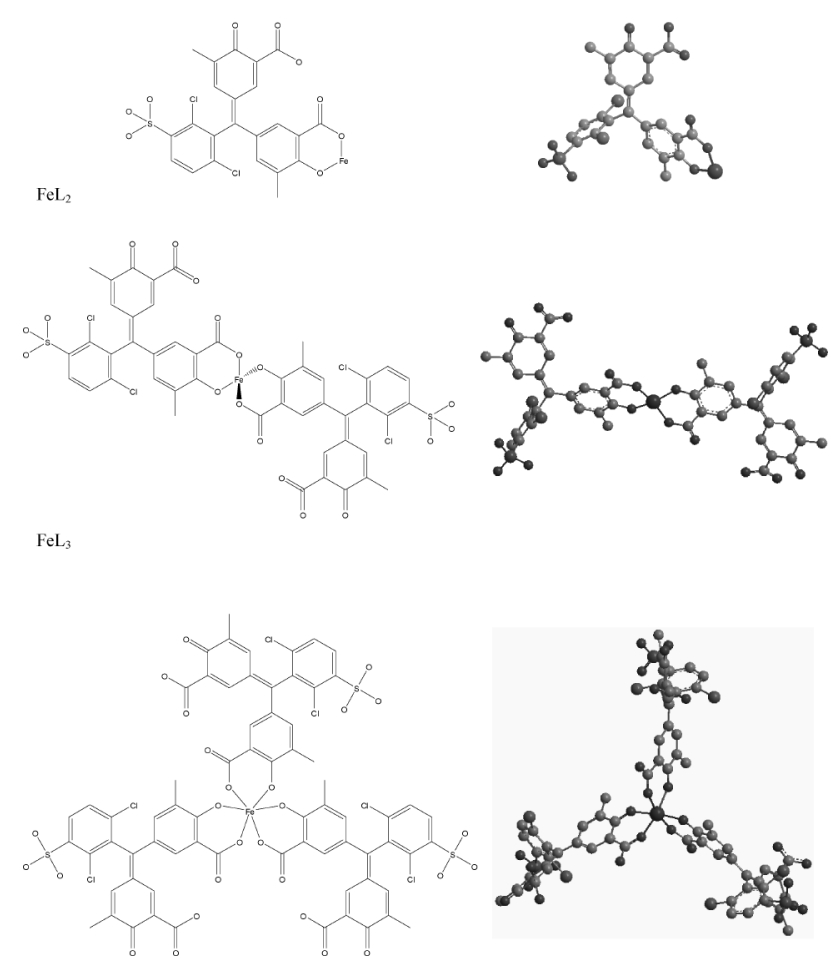

Fig. 8 The molecular structures of Fe-C.I. Mordant Blue 29 complexes with the composition $\mathrm{FeL}, \mathrm{FeL}_{2}$ and $\mathrm{FeL}_{3}$.

allowing stronger charge-transfer interactions between the longchain hydrophobic group of the cationic surfactant and the micelles of the Tween. The interaction between cationic and nonionic surfactants strongly depends on the hydrophobicity of the alkyl chain of the cationic surfactant. With increasing chain length, the interaction between the surfactants is enhanced. This leads to a decrease in the strength of the interactions between the cationic surfactant and the anionic dye, resulting in a decrease in the molar absorptivity of the ternary system.

The interactions between the $\mathrm{HL}^{3-}$ form of C.I. Mordant Blue 29 and the surfactants influence the formation of chelate complexes with iron(III). Investigations of ternary and quaternary complexes of iron(III) with C.I. Mordant Blue 29 
have shown that, in the presence of the surfactants, higher molar absorption coefficients are obtained as compared with the values for the corresponding binary chelate Fe-C.I. Mordant Blue 29 $\left(\varepsilon=0.43 \times 10^{5}-0.71 \times 10^{5} \mathrm{~L} \mathrm{~mol}^{-1} \mathrm{~cm}^{-1}\right)$. High molar absorption coefficients are connected with a higher degree of delocalization of the electron pair of the oxygen into the $\pi$-electron system of the ternary and quaternary complexes of iron(III). Furthermore, the increased molar absorption coefficients are also connected with the formation of dye-rich complexes.

\section{Acknowledgements}

The Gaussian 09 calculations were carried out at the ACK CYFRONET AGH, Kraków, Poland, under computational Grant No. MNiSW/SGI3700/UŚląski/102/2008.

\section{References}

1. S. Tunç and O. Duman, Fluid Phase Equilib., 2007, 251, 1.

2. F. Ahmadi, M. A. Daneshmehr, and M. Rahimi, Spectrochim. Acta, Part A, 2007, 67, 412.

3. H. Akbaş and T. Taner, Spectrochim. Acta, Part A, 2009, 73, 150.

4. B. Simončič and J. Špan, Dyes Pigm., 1998, 36, 1.

5. B. Simončič and M. Kert, Dyes Pigm., 2008, 76, 104.

6. A. Navarro and F. Sanz, J. Colloid Interface Sci., 2001, 237, 1.

7. H. Akbaş and C. Kartal, Spectrochim. Acta, Part A, 2005, $61,961$.

8. B. Gohain and R. K. Dutta, J. Colloid Interface Sci., 2008, 323, 395.

9. M. Jarosz and Z. Marczenko, Chem. Anal. [Warsaw], 1992, 37, 63.

10. K. Hayashi, Y. Sasaki, S. Tagashira, and E. Kosaka, Anal. Chem., 1986, 58, 1444.

11. Z. Marczenko and M. Balcerzak, "Separation, Preconcentration and Spectrophotometry in Inorganic Analysis", 2000, Elsevier, Amsterdam.
12. R. P. Pantaler and I. V. Pulyaeva, J. Anal. Chem., 1980, 35, 1021.

13. C. C. J. Roothan, Rev. Mod. Phys., 1951, 23, 69.

14. T. Helgaker, P. Jørgensen, and J. Olsen, "Molecular Electronic Structure Theory", 2000, Wiley, Chichester.

15. N. B. Balabanov and K. A. Peterson, J. Chem. Phys., 2005, 123, 064107.

16. M. J. Frisch, G. W. Trucks, H. B. Schlege, G. E. Scuseria, M. A. Robb, J. R. Cheeseman, G. Scalmani, V. Barone, B. Mennucci, G. A. Petersson, H. Nakatsuji, M. Caricato, X. Li, H. P. Hratchian, A. F. Izmaylov, J. Bloino, G. Zheng, J. L. Sonnenberg, M. Hada, M. Ehara, K. Toyota, R. Fukuda, J. Hasegawa, M. Ishida, T. Nakajima, Y. Honda, O. Kitao, H. Nakai, T. Vreven, J. A. Montgomery, Jr., J. E. Peralta, F. Ogliaro, M. Bearpark, J. J. Heyd, E. Brothers, K. N. Kudin, V. N. Staroverov, R. Kobayashi, J. Normand, K. Raghavachari, A. Rendell, J. C. Burant, S. S. Iyengar, J. Tomasi, M. Cossi, N. Rega, N. J. Millam, M. Klene, J. E. Knox, J. B. Cross, V. Bakken, C. Adamo, J. Jaramillo, R. Gomperts, R. E. Stratmann, O. Yazyev, A. J. Austin, R. Cammi, C. Pomelli, J. W. Ochterski, R. L. Martin, K. Morokuma, V. G. Zakrzewski, G. A. Voth, P. Salvador, J. J. Dannenberg, S. Dapprich, A. D. Daniels, Ö. Farkas, J. B. Foresman, J. V. Ortiz, J. Cioslowski, and D. J. Fox, Gaussian 09, Revision A.02, Gaussian, Inc., Wallingford CT 2009.

17. A. P. Martynov, V. P. Novak, and B. E. Reznik, J. Anal. Chem., 1977, 32, 519.

18. K. Ueno, T. Imamura, and K. L. Cheng, "Handbook of Organic Analytical Reagents", 1992, CRC Press, Boca Raton.

19. B. Simončič and M. Kert, Dyes Pigm., 2002, 54, 221.

20. M. Bielska, A. Sobczyńska, and K. Prochaska, Dyes Pigm., 2009, 80, 201.

21. K. R. Acharya, S. C. Bhattacharya, and S. P. Moulik, J. Photochem. Photobiol., A, 1997, 109, 29.

22. F. J. Langmyhr and K. S. Klausen, Anal. Chim. Acta, 1963, $29,149$.

23. D. Gotzmannová and V. Kubáň, Collect. Czech. Chem. Commun., 1980, 45, 1793.

24. V. Kubáň and D. Gotzmannová, Collect. Czech. Chem. Commun., 1980, 45, 2656. 